\title{
POD GENERATED BY MONTE CARLO SIMULATION USING A META-MODEL BASED ON THE SImSUNDT SOFTWARE
}

\author{
G. Persson, P. Hammersberg, and H. Wirdelius \\ Department of Materials and Manufacturing Technology, \\ Chalmers University of Technology, 41296 Göteborg, Sweden
}

\begin{abstract}
A recent developed numerical procedure for simulation of POD is used to identify the most influential parameters and test the effect of their interaction and variability with different statistical distributions. With a multi-parameter prediction model, based on the NDT simulation software simSUNDT, a qualified ultrasonic procedure of personnel within Swedish nuclear power plants is investigated. The stochastical computations are compared to experimentally based POD and conclusions are drawn for both fatigue and stress corrosion cracks.
\end{abstract}

Keywords: NDE Simulation, Simulated POD, Stochastic, simSUNDT, UT-01

PACS: $81.70 . \mathrm{Cv}, 43.35 . \mathrm{Zc}$

\section{INTRODUCTION}

With an aging industrial infrastructure, reliable maintenance and minimized unscheduled maintenance operations will be a very important challenge in the future. Another challenge concerns the extension of the in-service life of components while increasing the level of safety; this can only be achieved by a predictive maintenance. In order to quantify the inspection reliability the methodology of probability of detection (POD) was developed by the aeronautical industry in the early 1980's [1]. This statistical tool reduces the number of artificially produced artifacts that needs to be introduced into the test blocks in order to get statistically valid information of the detection capacity.

The European nuclear industry instead decided in the middle 1990's to develop an industry specific methodology in order to assess the NDT capacity. This ENIQ (European network of inspection qualification) approach intends to qualify not only the capability in detection but also in terms of sizing and characterization capabilities [2]. Much of the ENIQ approach is characterized by a documentation known as the Technical Justification of a qualification. This document specifies retrieved evidences (experimental data, physical reasoning or by modeling) of the system capacity.

Almost all qualification methodologies have been empirically based with extensive experimental work on test pieces. An infinite number of variables and possibilities then have to be reduced into a limited group of statistically relevant NDT situations [3]. Besides the problem of reconstructing the geometry and material, the fabricated defects also have to be introduced with a verified prescription of their sizes and NDT characteristics. In the recent decade a number of mathematical models have been developed and used as tools within parts of these qualification processes. 
A number of ongoing projects [4,5] address the possibility to enable simulated data to be used within the development of POD curves. One possible approach is then to have an optimized experimental phase (representative samples, but easy to manufacture) combined with much more efficiently retrieved simulated data. This study address the development of a procedure of stochastic simulations of POD curves based on synthetic produced data. These are retrieved by fitting a multi-parameter prediction model to the NDT simulation software simSUNDT [6]. The validation of this developed methodology for POD generation is based on a qualified ultrasonic procedure dating from 1996. This procedure, UT-01 [7], specifies manual ultrasonic inspection of piping components within Swedish nuclear power plants. A large number of inspectors have been qualified according to this procedure and their results (i.e. in detection) have also been used in a study with the ambition to estimate subsequent POD curve [8].

These POD curves are in this paper compared to corresponding curves generated with the above described simulation based methodology. It was feasible to identify the most influential parameters and to test the effect of their interaction and variability with different distributions. The dissimilar distributions of tilt and skew angles for real fatigue and stress corrosion cracks that were identified within the study have physical explanations. In this study it was possible to identify statistical distributions that enabled reconstruction of experimentally based POD curves.

\section{THE simSUNDT SOFTWARE}

The simSUNDT program is a Windows $®$-based pre and post-processor together (see Fig. 1) with a mathematical kernel (UTDefect, [9-12]) dealing with the actual

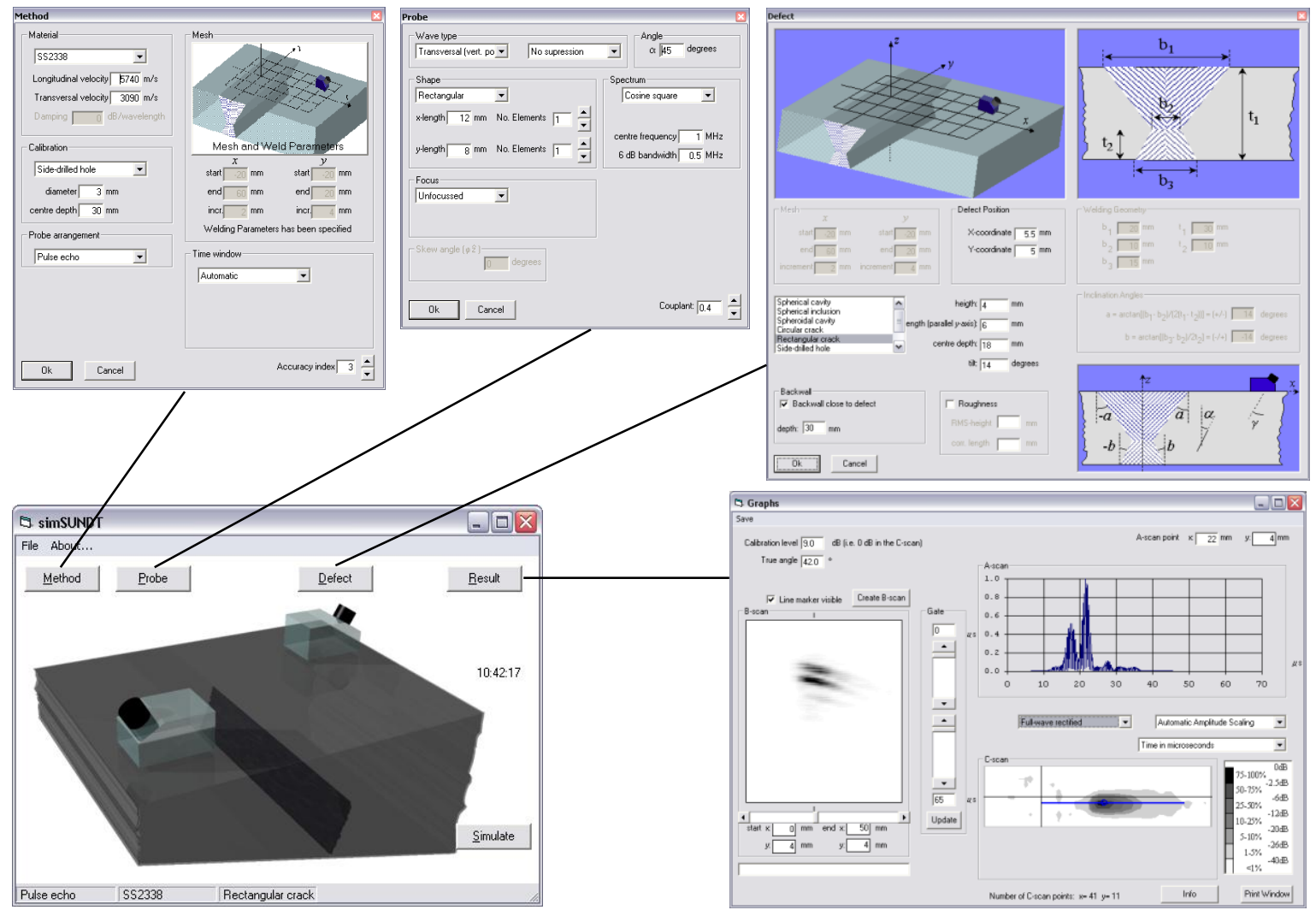

FIGURE 1. An overview of the pre- and postprocessor simSUNDT. 
mathematical modelling. The UTDefect computer code has been developed at the Dept. of Mechanics at Chalmers University of Technology and has been experimentally validated and verified [10-12]. The model employs various integral transforms and integral equation techniques to model probes and the scattering by defects. The software simulates the whole testing procedure with the contact probes (of arbitrary type, angle and size) acting in pulse-echo or tandem inspection situations.

The simulated test piece in the present study is homogeneous and of an isotropic material. The component is two dimensional, i.e. an infinite plate with finite thickness $(t)$, bounded by the scanning surface where one probe is scanning the object and a planar back surface as shown in Fig. 2.

The probe is modelled as a circular single crystal shear wave transducer, used in a pulse echo inspection situation. The probe is unfocused with a cosine square frequency spectrum, probe angle $\gamma$, and the contact medium (i.e. the boundary condition) is chosen to correspond to gel. All parameters are set to model the conventional probes MSWQC45 according to Table 1. The calibration defect is a side-drilled hole (SDH) of diameter 2.4 $\mathrm{mm}$ and in order to simulate DAC-calibration, the SDH is placed at the same depth as the defect.

The defect model is a surface breaking strip like crack, of height $a$. Even though it is a two dimensional defect it includes a possibility to model both skew and tilt $(\alpha)$ angle [13].

The simSUNDT model has been validated for the actual simulated situation in an ultrasonic benchmark study initiated by the World Federation of NDE Centers [14]. The experiments included experimentally measured signal responses of side-drilled holes and rectangular surface breaking defects in dimensions that are covered by Tables 1 and 2 . The agreement found, between simulations and experiment, was very good for the simSUNDT model.

\section{THE UT-01 EXPERIMENTAL ULTRASONIC PROCEDURE}

The UT-01 procedure specifies manual ultrasonic inspection of piping components within Swedish nuclear power plants and cover techniques for detection, sizing and characterization. It covers both fatigue cracks and intergranular stress corrosion cracks (IGSCC). The procedure specifies in details parameters such as; components, defects, level of competence (personnel), method, equipment, calibration and inspection procedure with e.g. detection criteria, characterization procedure and techniques for sizing of defects.

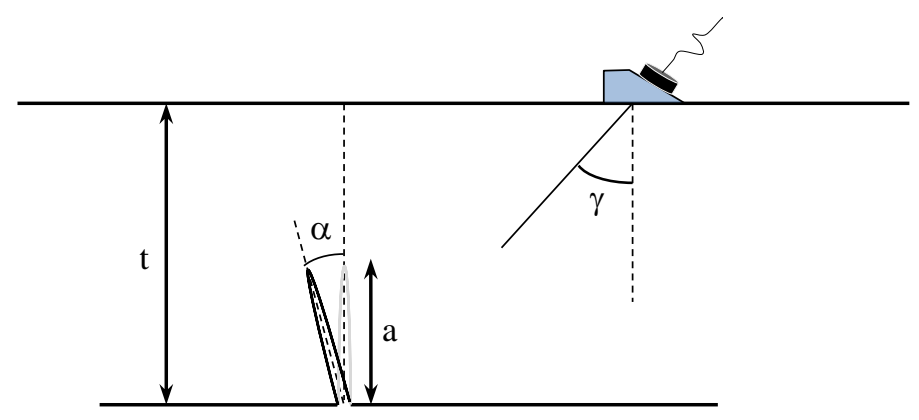

FIGURE 2. The geometry of the ultrasonic pulse echo situation with a surface breaking crack. 
Based on the procedure all identifiable parameters are subdivided into influential and essential parameters according to the definition:

- Influential parameters can potentially influence the outcome of an inspection.

- Essential parameters are those influential parameters whose change in value would actually affect a particular inspection in such a way that the inspection could no longer meet its defined objectives.

The latter have to be addressed within the Technical Justification with a subdivision into Set 1 and Set 2 parameters. Essential parameters that are identified as Set 1 must be specified with correlating uncertainties and their distribution. Based on the $U T$ 01 documentation [7], the corresponding Technical Justification [15] and restraining to parameters that are available within the simSUNDT software it was possible to identify the essential parameters defined in Table 1 and 2. In this study the defect size/height is treated as a Set 2 parameter since it is a control factor. It was identified within the report that the wall thickness did not seem to be a significant parameter in itself. This can be explained by the procedure since applied calibration, distance amplitude correction (DAC), intends to withdraw this is as an essential parameter.

In order to quantify the capability of the UT-01 procedure data from the qualifications during the period 1996 to 2004 was composed in a project [8]. Used test pieces within the qualification were of austenitic stainless steel piping and the wall thickness varied between 4 and $35 \mathrm{~mm}$. The defects consisted of both artificial defects and implanted defects from both fatigue and stress corrosion. Results from investigation of 97 cracks from 55 different test cases involving 41 different ultrasonic technicians (level II according to EN 473) were used for the estimation of the POD curves.

When only stress corrosion cracks (IGSCC) were considered, the POD was estimated in the report [3] as;

$$
P O D=\Phi(0.1218+0.3720 \cdot \ln (a))
$$

where $\Phi$ denotes the normalised Gaussian distribution.

When only the fatigue cracks were considered, a significant higher detection rate was revealed and the estimated POD function was estimated as;

$$
P O D=\Phi(0.6503+0.3720 \cdot \ln (a))
$$

Notable is that the benchmark [14] included the conventional circular 45 degree shear wave contact transducer MSWQC45 which was used within the UT-01 procedure in the investigation [8].

\section{THE SIMULATION PROCEDURE}

The methodology developed and described in [13] is here used to emulate realistic POD curves. The ultrasonic simulation software simSUNDT is used as kernel for the stochastical computations. The technique of building a meta-model (response surface) for the stochastic simulation starts with identifying the essential parameters. Based on the 
TABLE 1. The various distributions of essential Set 1 parameters represented in Fig. 2.

(*nominal angle $48^{\circ}$ and $66^{\circ}$ respectively).

\begin{tabular}{|l|l|l|c|c|c|}
\hline Stochastic distributed parameters & Note & Distribution & Mean & Delta & SD \\
\hline Defect tilt angle $(\alpha)$ & Crack type IGSCC & Uniform & 0 & 15 & \\
\hline Defect tilt angle $(\alpha)$ & Crack type Fatigue & Normal & 0 & & 6.67 \\
\hline Defect skew angle & Crack type IGSCC & Uniform & 0 & 20 & \\
\hline Defect skew angle & Crack type Fatigue & Normal & 0 & & 10 \\
\hline Probe angle $(\gamma)^{*}$ & Mean according to UT-01 & Normal 45 & 45 & & 0.8 \\
\hline Probe angle $(\gamma)^{*}$ & & Normal 60 & 60 & & 1.1 \\
\hline Probe diameter & Mean according to UT-01 & Normal & 6.5 & & 0.32 \\
\hline
\end{tabular}

UT-01 documentation [7], the corresponding Technical Justification [15] and restraints to parameters that are available within the $\operatorname{sim} S U N D T$ software it was possible to identify the essential parameters for the mathematical modelling defined in Table 1. These parameters have a large influence of the response of the numerical model and are chosen according to Table 1. For the defect tilt and skew in Table 1 the uniform distribution is chosen as two standard deviations in width, and for the probe angle as one standard deviation.

Following the methodology we note that the morphological difference between fatigue cracks and stress corrosion cracks $[16,17]$, that the latter tends to be more tilted and often branched, is not possible to model here. However, fatigue cracks are normally perpendicular to the major stress direction and are typically straight with no branching. Stress corrosion cracks on other hand are considerable more dependent on the micro structure, and deviation from pure stress controlled growth is thus often observed. Hence there are physical explanations to why the distribution of tilt and skew angles for real fatigue and stress corrosion cracks may differ. Both crack types will here be modelled as a surface breaking strip like crack (simSUNDT). The latter since [8] make clear that the experimentally investigated cracks had lengths that were more than 4 times their heights and therefore essentially two dimensional. The data in Table 1 for the crack type fatigue and IGSCC (stress corrosion crack) is then chosen as

- The fatigue crack is prescribed to have a normal distribution of skew and tilt. The distribution was chosen with a mean of $0^{\circ}$ with two standard deviations in width for both the tilt and the skew angle (see Table 1).

- The IGSCC is prescribed to have a uniform distribution of skew and tilt angle, i.e. no preferred growth direction within the specified limits (see Table 1).

TABLE 2. The various essential Set 2 parameters represented in Fig. 2.

\begin{tabular}{|l|l|l|}
\hline Essential parameter & Status & Value \\
\hline Defect size/height $(\mathrm{a})^{*}$ & Control factor & $2-20 \mathrm{~mm}$ \\
\hline Object thickness $(\mathrm{t})^{*}$ & Reduced due to DAC & $35-50 \mathrm{~mm}$ \\
\hline Centre frequency & Constant & $2.25 \mathrm{Mhz}$ \\
\hline Bandwidth & Constant & $44 \%$ \\
\hline Couplant & Constant & 0.4 \\
\hline Wave speed $\left(C_{\mathrm{L}}\right)$ & Constant & $5720 \mathrm{~m} / \mathrm{s}$ \\
\hline Wave speed $\left(C_{\mathrm{S}}\right)$ & Constant & $3120 \mathrm{~m} / \mathrm{s}$ \\
\hline Density & Constant & $7950 \mathrm{~kg} / \mathrm{m}^{3}$ \\
\hline
\end{tabular}


As in the experimental POD study [8] the object thickness is reduced as an essential parameter by the calibration procedure within UT-01. The remaining Set 1 parameter, the defect size (a) is then a control factor when creating a meta-model, the Set 2 parameters are treated as constants.

Starting with a multi level full factorial sequence for the Set 1 parameters and combining this with a central composite design method (cubic face centred) eventually a total of 295 simulations were executed with $\operatorname{sim} S U N D T$. The response surface for the normalised DAC signal response was then created by surface fitting of Hardy's MultiQuadratics radial basis functions to the simulated values. The stochastic simulation was made by using a Latin hypercube sampling (LHS) for creation of 5000 random designs for each data set, data according to Table 2.

Figure 3 shows the POD based on the UT-01 detection criterion, that is a signal response above $50 \%$ of received amplitude from the reference defect (i.e. $2.4 \mathrm{~mm}$ side drilled hole at back wall depth). As in [13] the POD for the numerical simulation is measured as the percentage of the 5000 simulated signal responses above $-6 \mathrm{~dB}$. The different POD curves in the figure represent different distribution of tilt and skew angles according to Table 1, compared to experimentally estimated POD [8] for fatigue cracks and IGSCC using UT-01.

The simulation for the $45^{\circ}$ probe, as predicted by UT-01, shows a very good agreement between the refined meta-model and the experimental based POD. Here one should remember that the valid limit of $U T-01$, based on equations 1 and 2, is $2 \mathrm{~mm}$ since there were no defects less than this size among the qualification defects. Figure 3 also show that it is possible to identify different distributions of skew and tilt angles defining the defects as being fatigue or IGSCC in an ultrasonic signal response perspective.

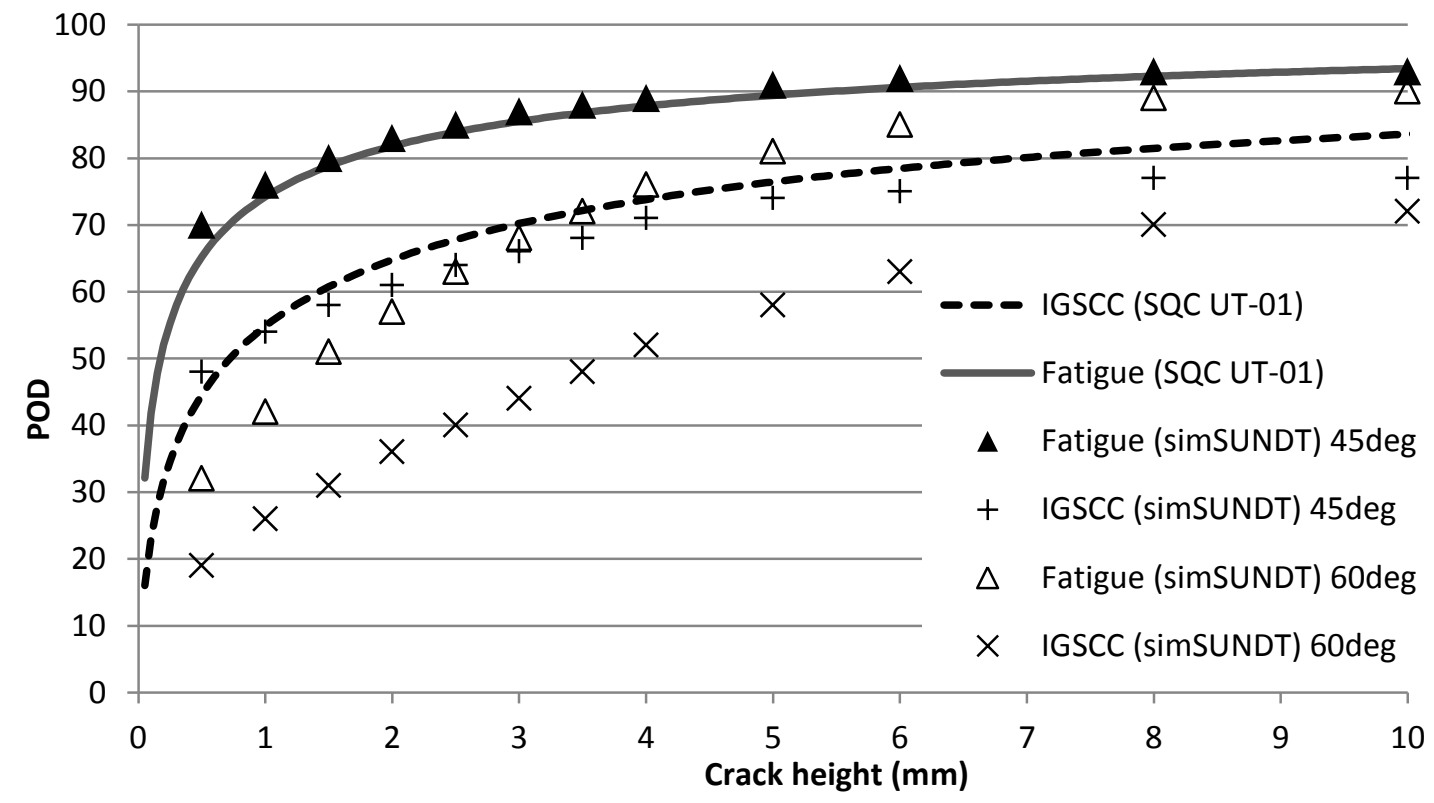

FIGURE 3. Experimentally estimated POD (eq. 1 and 2) compared with POD evolved by simulations (two different probe angles $45^{\circ}$ and $60^{\circ}$ ). 

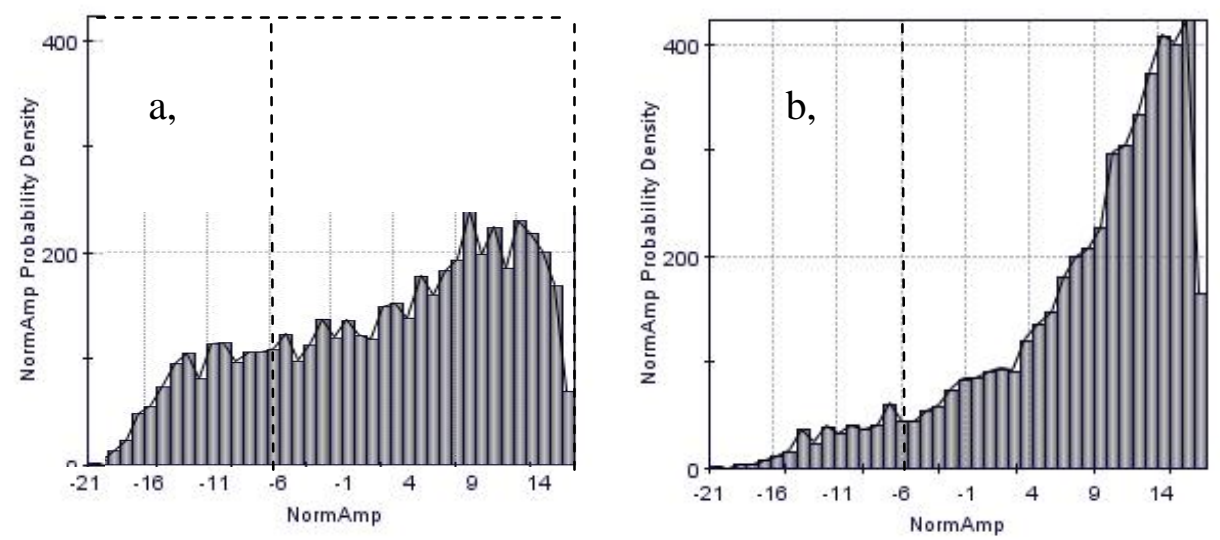

FIGURE 4. Distribution of experimentally DAC-normalised signal response for a surface breaking crack of height $8 \mathrm{~mm}$. Fatigue crack to the left and IGSCC to the right. Detection level (-6dB) indicated in figure. things

Using the statistical data for the $60^{\circ}$ probe, according to Table 1 , shows three

- The POD is drastically reduced for both the fatigue crack and the IGSCC, that is the simulation verifies that the $U T-01$ prescribed $45^{\circ}$ is a better choice than a 60 probe.

- There is still possible to distinguish between the two crack types, given that the same probe is used in both cases.

- For large cracks the POD seems to be about the same for both the $45^{\circ}$ and the $60^{\circ}$ probe. This makes sense since if the crack is large enough it will be detected regardless of the probe angle.

It is also possible to study the probability density, Fig. 4 shows the distribution of the DAC-normalised signal response for a surface breaking crack of height $8 \mathrm{~mm}$. Fig. $4 \mathrm{a}$ is for the fatigue crack and Fig. $4 \mathrm{~b}$ for the IGSCC, data according to Table 1, both figures are computed for the $45^{\circ}$ probe. Clearly the signal response for both defects deviates from a normal distribution, this is a result of the higher order behaviour of the response-surface. In this case a fatigue crack yields a more narrow density distribution than the IGSCC. Further numerical investigations for normal distributions have shown that a higher standard deviation gives a more narrow signal response. In both figures the $-6 \mathrm{~dB}$ detection level is indicated.

\section{CONCLUDING REMARKS}

The presented multi-parameter prediction model shows a good agreement when comparing to experimentally based $\operatorname{POD}(a)$. The results show that the statistical distributions for the influential parameters, such as crack tilt and skew, is essential when comparing fatigue and stress corrosion cracks. Moreover it seems like macroscopic branching and surface roughness is of less importance when reconstructing the experimentally based POD.

Computations verifies that using the qualification procedure UT-01 there should be very little risk to confuse fatigue and stress corrosion cracks from a POD(a) perspective. The simulation also verifies that the $45 \mathrm{deg}$ probe used in UT-01 is a good choice, computations for a corresponding $60 \mathrm{deg}$ probe yields a less probability of detection. 
Furthermore, the stochastic computations have shown that the construction of the meta-model is of great importance. The points used to create the meta-model (response surface), given by numerical computation with $\operatorname{sim} S U N D T$, have to be chosen wisely due to their large impact on the model's behaviour. This is believed to be a result of the very intricate multi-parameter response surface.

Notable is also that even if the used statistical distributions for the influential parameters are assumed uniform or normal, the distribution of the signal response (POD) is deviant from this. This is another result of the higher order response surface.

\section{REFERENCES}

1. "Nondestructive Evaluation System Reliability Assessment", MIL-HDBK-1823A (2009)

2. ENIQ Recommended Practice 2: "Recommended Contents for a Technical" Justification, http://safelife.jrc.ec.europa.eu/eniq/publications/complete.php

3. H. Wirdelius, "Examples of mathematical modeling used as a tool within NDT qualification", 1 Int. Conf. NDE in relation to structural integrity for nuclear and pressurised components, Amsterdam (1998).

4. Model Assisted POD working group, http://www.cnde.iastate.edu/MAPOD

5. F. Jensen, S. Mahaut, P. Calmon, and C. Poidevin, "Simulation based POD evaluation of NDI techniques", $10^{\text {th }}$ European conference on non-destructive testing, Moscow (2010).

6. G. Persson and H. Wirdelius, "Recent survey and application of the simSUNDT software", QNDE 2009, AIP Conference Proceedings, 1211 s. 2125-2132

7. C. Ericson, "Provningsprocedur för manuell ultraljudsprovning av rör och komponenter”, UT-01 rev2, Swedish Nuclear Power Plant Owners, (2010).

8. T. Jelinek, L. Tidström, and B. Brickstad, "Probability of Detection for the Ultrasonic Technique according to the UT-01 Procedure”, SKI Report 2005:03, Stockholm (2005).

9. A. Boström, SKI Report 95:53, Stockholm (1995).

10. A. S. Eriksson, A. Boström, and H. Wirdelius, SKI Report 97:1, Stockholm (1997).

11. P. Bövik and A. Boström, "A model of ultrasonic non-destructive testing for internal and subsurface cracks", J. Acoust. Soc. Am. 102, pp. 2723-2733 (1997).

12. H. Wirdelius, "Experimental validation of the UTDefect simulation software", Proc. 6th Int. Conf. on NDE in Relation to Structural Integrity for Nuclear and Pressurized Components, Budapest (2007).

13. H. Wirdelius and G. Persson, "Simulation Based Validation of the Detection Capacity of an Ultrasonic Inspection Procedure", International Journal of Fatigue (In press)

14. L. Schmerr, 2009 Ultrasonic benchmarks Part II - problems, (http://www.wfndec.org)

15. M. Strand, "Teknisk motivering Projekt UT01”, ÅF Report 505179-RAPP-01, (2010).

16. P. Ekström and J. Wåle, "Characterisation for In-Service Inspection Planning", SKI Report 95:70, Stockholm (1995).

17. J. Wåle, "Characterisation for In-Service Inspection Planning an Update", SKI Report 2006:24, Stockholm (2006). 